\title{
Phase-Averaged Method Applied to Periodic Flow Between Shrouded Corotating Disks
}

\author{
Shen-Chun Wu \\ Department of General Education, National Defense University, Taoyuan, Taiwan, Republic of China \\ Yau-Ming Chen \\ Department of Mechanical Engineering, National Taiwan University, Taipei, Taiwan, Republic of China
}

This study investigates the coherent flow fields between corotating disks in a cylindrical enclosure. By using two laser velocimeters and a phase-averaged technique, the vortical structures of the flow could be reconstructed and their dynamic behavior was observed. The experimental results reveal clearly that the flow field between the disks is composed of three distinct regions: an inner region near the hub, an outer region, and a shroud boundary layer region. The outer region is distinguished by the presence of large vortical structures. The number of vortical structures corresponds to the normalized frequency of the flow.

Keywords Corotating disks, Laser doppler velocimetry, Phaseaveraged method

The fluid motion in the space between a pair of corotating disks is of considerable importance to hard disk drives, which are extensively used as data-storage devices in computers. This is because the large-scale oscillating flow structure induced by the rotation of disks and the vortexes being shed by obstructions contribute to alignment inaccuracies and, hence, to degraded performance by the read-write magnetic heads that are supported at a submicron distance from the rotating disk surfaces.

Lennemann (1974) visualized the flow in an axisymmetrical cylindrical shroud by using aluminum powder as the tracer. Without an obstruction, Lennemann observed a laminar solid body rotation core region with an unsteady polygonal, lobedshaped boundary. The core was reported to rotate at $80 \%$ of the disk rotation rate, and highly turbulent flow was observed

Received 31 May 2002; accepted 31 May 2002.

Address correspondence to Professor Yau-Ming Chen, Department of Mechanical Engineering, National Taiwan University, Taipei, Taiwan 106, R.O.C. E-mail: ymchen@ccms.ntu.edu.tw near the shroud. Using a similar water flow apparatus and visualization technique, Kaneko and colleagues (1977) observed a laminar core that extended from the hub out to the middle radii of the disks. An outer turbulent region surrounded the bumpy laminar core. Abrahamson and colleagues $(1988,1989,1991)$ employed bromothymol blue dye and a hydrogen bubble technique for flow visualization and reported the existence of a center core region in solid body rotation and the presence of circumferentially periodic flow structures with a strong component of axial vorticity near the shroud, processing at roughly $75 \%$ of the disk rotation rate.

Schuler and colleagues (1990) employed laser Doppler velocimetry (LDV) to measure the mean and root mean square (rms) of the circumferential velocity component between the central pair of four corotating disks. Normalized with the local disk surface velocity, the mean and rms velocity measured by Tzeng and colleagues (1991) at $\operatorname{Re}=7.9 \times 10^{4}$ show fairly good agreement with the data measured by Schuler and colleagues at $\operatorname{Re}=8.9 \times 10^{4}$. The time sequence measured by Schuler and colleagues displays distinct sinusoid-like oscillations. By using a copper-vapor laser for illuminating aerosol flow between corotating disks and employing a high-resolution Change Coupled Device (CCD) camera, Tzeng and colleagues (1991) were able to report the number of sides of the polygonal interface at high disk speeds, and they confirmed the agreement of the inscribing radius of the polygonal structure, evaluated by flow visualization.

Tzeng and Humphrey (1991) used LDV to measure the mean and rms of the circumferential velocity components between corotating disks in an axisymmetrical enclosure. Their normalized radial profiles of the circumferential mean velocity showed two flow regions separated by a dimensionless radius $r^{*}=0.55$. These consist of an inner region $\left(r^{*}<0.55\right)$ where the fluid velocity is within $10 \%$ of the disk velocity and an outer region $\left(r^{*}>0.55\right)$ where the normalized velocity decreased linearly to $\cong 63 \%$ of the disk velocity at the disk edge $\left(r^{*}=1\right)$. 
By illuminating the microsized mineral oil droplets with a laser sheet, Humphrey and Gor (1993) visualized the crossstream flow in an experimental apparatus similar to that used by Schuler and colleagues (1990). Humphrey and colleagues (1995) numerically investigated the unsteady streamlined motion of a constant property fluid in the unobstructed space between disks corotating in a fixed cylindrical enclosure using a time-explicit algorithm.

Although efforts to record the experimental measurements were made in the above-mentioned studies, the unsteady aspects of the oscillatory flow structure induced by shrouded corotating disks are still not well understood. Previous studies focused on the flow visualization methods; quantitative measurement data are, therefore, still lacking. So, in this study, a measuring technique employing two LDVs together with a phase-averaged method was developed to accomplish time-resolved measurements of periodic flow structures induced by shrouded corotating disks.

\section{EXPERIMENTAL APPARATUS AND TECHNIQUES}

\section{Apparatus and LDV Instrumentation}

The experimental test section and its associated coordinate system are shown schematically in Figure 1. Two rigid disks with an outer radius of $R_{2}=187.5 \mathrm{~mm}$ were concentrically mounted on a hub. The disks were made of transparent Plexiglas, thickness $b=10 \mathrm{~mm}$. A spacer ring, $h=23.2 \mathrm{~mm}$ and radius $R_{1}=59.4 \mathrm{~mm}$ was used to separate the disks in the direction of the spindle axis (z). The test section shroud was made of transparent Plexiglas of a rectangular parallelepiped outer shape and was machined to achieve a cylindrically axisymmetric inner wall, radius $R_{2}+a=198.5 \mathrm{~mm}$. The base and the top cover of the test section were also made of Plexiglas so as to allow the passage of laser light. Pure water was used as the working fluid in the experiment. Particles of magnesium oxide with a diameter of about $1.2 \mu \mathrm{m}$ were used as seeding material for the LDV measurements.

The principles and practice of laser Doppler anemometry have been introduced elsewhere (e.g., Durst et al., 1981). Only the LDV system with direct relevance to this study has been mentioned here. Two laser velocimeters, LDV and Phase Doppler Particle Analyzer (PDPA), were used in the present work in order to carry out the measurements using a phase-averaged technique. The optics and electronic setup are schematically shown in Figure 2. The TSI Model 9201 Colorburst Multicolor Beam Separator converts an argon-ion laser beam (Coherent Innova $90,2 \mathrm{~W}$ ) into four laser beams to perform LDV. The conversion produces two pairs of shifted and unshifted output beams: two green beams at $514.5 \mathrm{~nm}$, and two blue beams at $488.0 \mathrm{~nm}$. A Bragg cell was used to shift the frequency of each color to

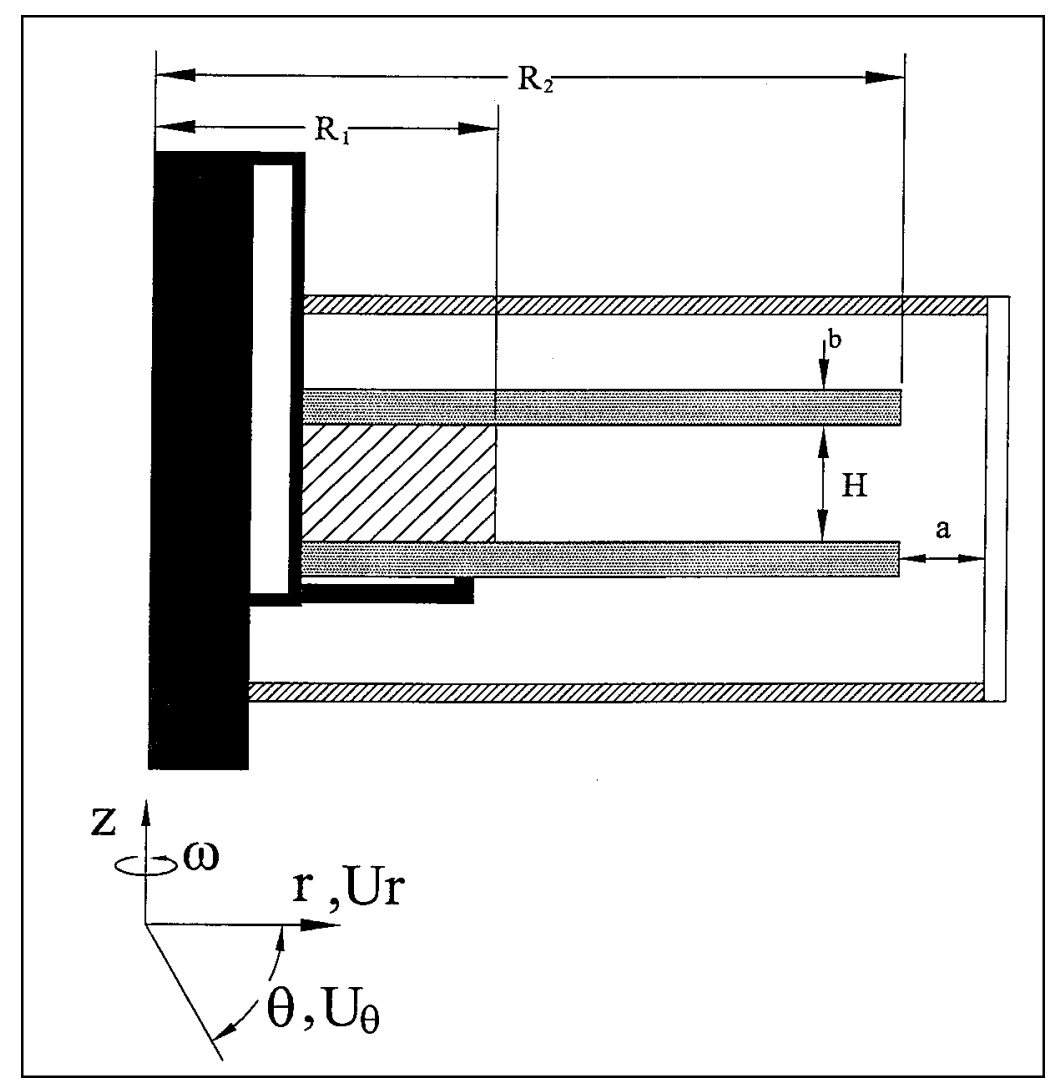

FIGURE 1

Test section and coordinate system. 


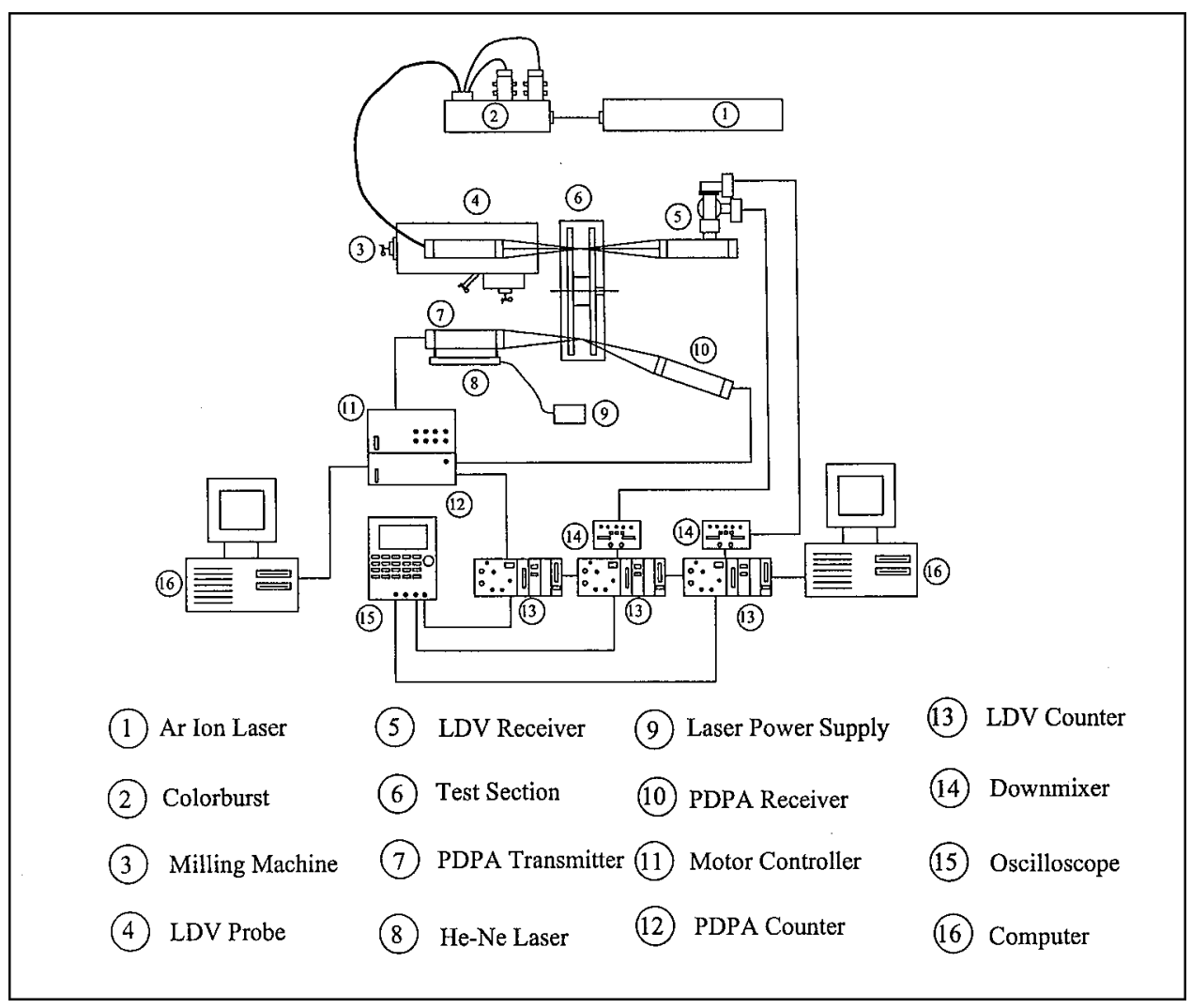

FIGURE 2

LDV systems.

$40 \mathrm{mHz}$ so as to eliminate directional ambiguity. The Model 9271 Coupler launches light into a single-mode polarizationpreserving fiber. The resulting two pairs of beams were then passed through a $350-\mathrm{mm}$ focal length lens. The focused beams intersected between the pair of disks, producing a probe volume with the dimension $0.52 \mathrm{~mm} \times 0.10 \mathrm{~mm}$ at the $\left(\mathrm{e}^{-2}\right)$ extent of light intensity. The light scattered from the seeding particles was collected by a receiving optical package, which reflects the collected light into a photomultiplier. The detected signal was electrically downmixed to the approximate frequency shift $(0.1 \mathrm{mHz} \sim 10 \mathrm{mHz}$ in this experiment). TSI counter processors with 1-nsec resolution were then used to process the Doppler signals. The Doppler signals were monitored on an oscilloscope, and the digital output of the counter processors was fed directly into a personal computer for storage and further analysis.

The PDPA system consists of five major components: transmitter, receiver, signal processor, motor controller box, and computer. The Aerometrics model XMT-1100-4S transmitter was used to generate two laser beams of equal intensity and focus them on an intersection point, which formed the measurement region. The transmitter contains a Spectra-Physics model SP-106, $10 \mathrm{~mW}$, polarized helium-neon laser. Optical components within the transmitter serve to focus, partition, and collimate the laser beam. A radial diffraction grating beam splitter was mounted on the shaft of a high-speed direct-current motor. Rotation of the grating produces a frequency differential between the diffracted beams, which is used to produce a frequency shift in the measured particle signals. The receiver model RCA- 2100 collected the light scattered from seed particles. The receiver was then connected to the PDP-3100 signal processor with $2 \mu \mathrm{sec}$ resolution to process the Doppler signals. The ana$\log$ output of PDP-3100 signal processor was transmitted to the TSI counter processor as input to offer the phase reference.

\section{Measurement Technique and Data Reduction}

The rotational speed of the disks was fixed at $266 \mathrm{rpm}$ in this study. By using the radius of the disk as the characteristic length and the disk tip speed as the reference velocity, the Reynolds number was calculated to be about $\operatorname{Re}=1.06 \times 10^{6}$. The dimensionless axial distance of disks was fixed at $S=0.12$ $\left(S=H / R_{2}\right)$. Experiments were also carried out at $S=0.05$ for comparison. As shown in Figure 2, the PDPA probe was fixed at a specific position $\left(r^{*}=0.72, z / H=0.5\right)$. To investigate the vortical structures, all the measurements were made in a quarter of the center plane between the two corotating disks. The grids in the measuring domain totaled 4096 points. As the measurements ran, the PDPA probe was kept at the specific position as a phase reference point, while the probe of the LDV was translated in the 
measurement domain by the transverser. Doppler burst signals detected from the PDPA and LDV were transferred to the LDV counters, which worked as a coincident mode under the control of the TSI MI-990 interface module. A coincident time window length $(0.5 \mathrm{msec})$ was set in the MI- 990 interface module to test the synchronization of burst signals detected by the PDPA and LDV. The burst signals were processed to compute the Doppler shift frequency, as the time interval of signals detected by PDPA and LDV was shorter than the coincident time window length. The Doppler shift frequency data were then transferred to a personal computer via a Direct Memory Access (DMA) interface card. The velocity values were further calculated, and then the time sequence was stored. The coincident data rate was kept at about $2000 \mathrm{~Hz}$ as the measurements were made, which was high enough to resolve the periodic motion of the vortical structure with $9.9 \mathrm{~Hz}$ of frequency. During each measurement, more than 20,000 samples of the time sequence of reference points (measured by PDPA) and measuring points were collected. All of the time histograms of velocity, whether circumferential or radial, were measured with respect to the same phase reference point. The phase-averaged method is shown schematically in Figure 3.
A cycle was built so there were two adjacent peak values in the phase reference signal; it was divided into 10 phases of the same time interval. The time series measured by LDV corresponding to each phase was sampled and averaged, respectively.

In order to investigate periodic flow fields, a decomposition of velocity proposed by Hussain and Reynolds (1970), Reynolds and Hussain (1972) was used in analyzing the organized wave in the turbulent shear flow. The oscillatory flow of corotating disks can be taken as combination of global mean components $\bar{u}$, a periodic mean $\tilde{u}$, and a random component $u^{\prime}$. By definition, the instantaneous velocity is the sum

$$
u=\bar{u}+\tilde{u}+u^{\prime}
$$

According to an analysis by Reynolds and Hussain (1972), the momentum equation that taking average at constant phase has the form

$$
\rho \frac{\partial\left\langle u_{i}\right\rangle}{\partial t}+\rho\left\langle u_{j}\right\rangle \frac{\partial\left\langle u_{i}\right\rangle}{\partial x_{j}}=-\frac{\partial\langle p\rangle}{\partial x_{i}}+\mu \frac{\partial^{2}\left\langle u_{i}\right\rangle}{\partial x_{k}^{2}}-\rho \frac{\partial\left(u_{i}^{\prime} u_{j}^{\prime}\right)}{\partial x_{j}}
$$

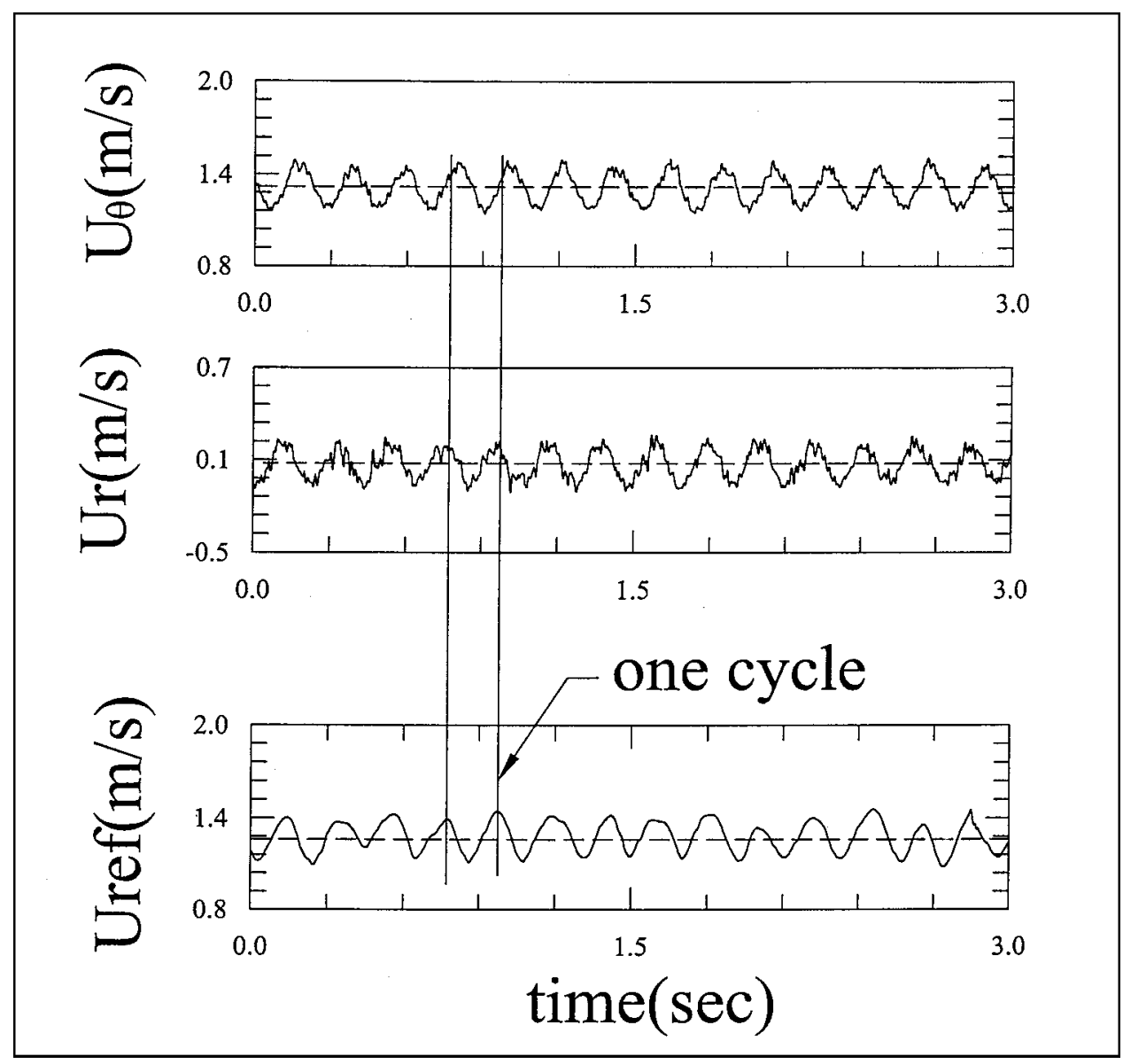

FIGURE 3

Typical time records of the circumferential and radial velocity at $r^{*}=0.395$. 
The momentum equation for the global mean flow has the form

$$
\rho \bar{u}_{j} \frac{\partial \bar{u}_{i}}{\partial x_{j}}=-\frac{\partial p}{\partial x_{i}}+\mu \frac{\partial^{2} \bar{u}_{i}}{\partial x_{k}^{2}}-\rho \frac{\partial\left(\overline{\left\langle u_{i}^{\prime} u_{j}^{\prime}\right\rangle}+\overline{\tilde{u}_{i} \tilde{u}_{j}}\right)}{\partial x_{j}}
$$

Because of space limitations, only the results of the global mean components are discussed here.

Any bias in calculating the global mean velocity was corrected by using the method provided by McLaughlin and Tiederman (1973). Calibration was carried out in the test section with an oscilloscope, and the maximum velocity bias was estimated to be less than $2 \%$. The statistical uncertainty for phaseaveraged velocity was estimated to be within $4.5 \%$; the typical value was about $1.2 \%$.

\section{RESULTS AND DISCUSSION}

\section{Mean Velocity Profiles}

Figure 4 shows the normalized mean circumferential velocity component measured as a function of the radial position along the symmetrical plane, midway between the two disks $(z / H=0.5)$. The mean values have been normalized by the local disk speed. A horizontal dashed line was plotted to represent the local disk speed $\mathrm{U} / \mathrm{r} \omega=1$. The dashed line manifests the solid body rotation. Figure 4 shows that the velocity of the fluid begins to deviate from the solid body rotation at the position of about $r^{*}=0.35$ in the present study. Included for comparison are also the results measured by Abrahamson and colleagues $(1989)\left(\operatorname{Re}=4.5 \times 10^{5}\right)$ and Tzeng and colleagues
(1991) $\left(\operatorname{Re}=7.9 \times 10^{4}\right)$. The circumferential velocity begins to deviate from the solid body rotation at nearly the same dimensionless radial position of about 0.45 in the results measured by Abrahamson (1989) and Tzeng and their colleagues (1991). The difference is caused by the larger axial distance of the disks in the present work. The dimensionless axial distance in the other studies was fixed at $S=0.05$, whereas the value adopted in this study was $S=0.12$. The axial distance of the disks in the studies of Abrahamson (1989) and Tzeng (1991) and their colleagues was so small as to prevent the inflow with momentum deficit from penetrating a greater distance inside. For comparison, the experiments were also performed at $S=0.05$. As can be seen, good agreement can be found between the present results and those of Abrahamson (1989) and Tzeng (1991) and their colleagues.

\section{Power Spectra}

Figure 3 shows the measured time-resolved circumferential and radial velocities at the midplane between the two disks at $r^{*}=0.395$. These time sequences display distinct sinusoid-like oscillations. The Fourier spectra of radial and circumferential velocities at several radial positions were calculated. The results of radial and circumferential velocity power spectra were presented as examples in Figures 5 and 6 . The actual frequencies of the spectra were normalized by 0.75 times the disk rotational frequency. The spectral results show peaks in energy at an integral normalized frequency of $f^{*}=3$. Abrahamson and colleagues (1989) reported similar results.

In addition to the spectral frequency, Figure 5 shows a plot of spectral intensity as a function of radial position. For radial

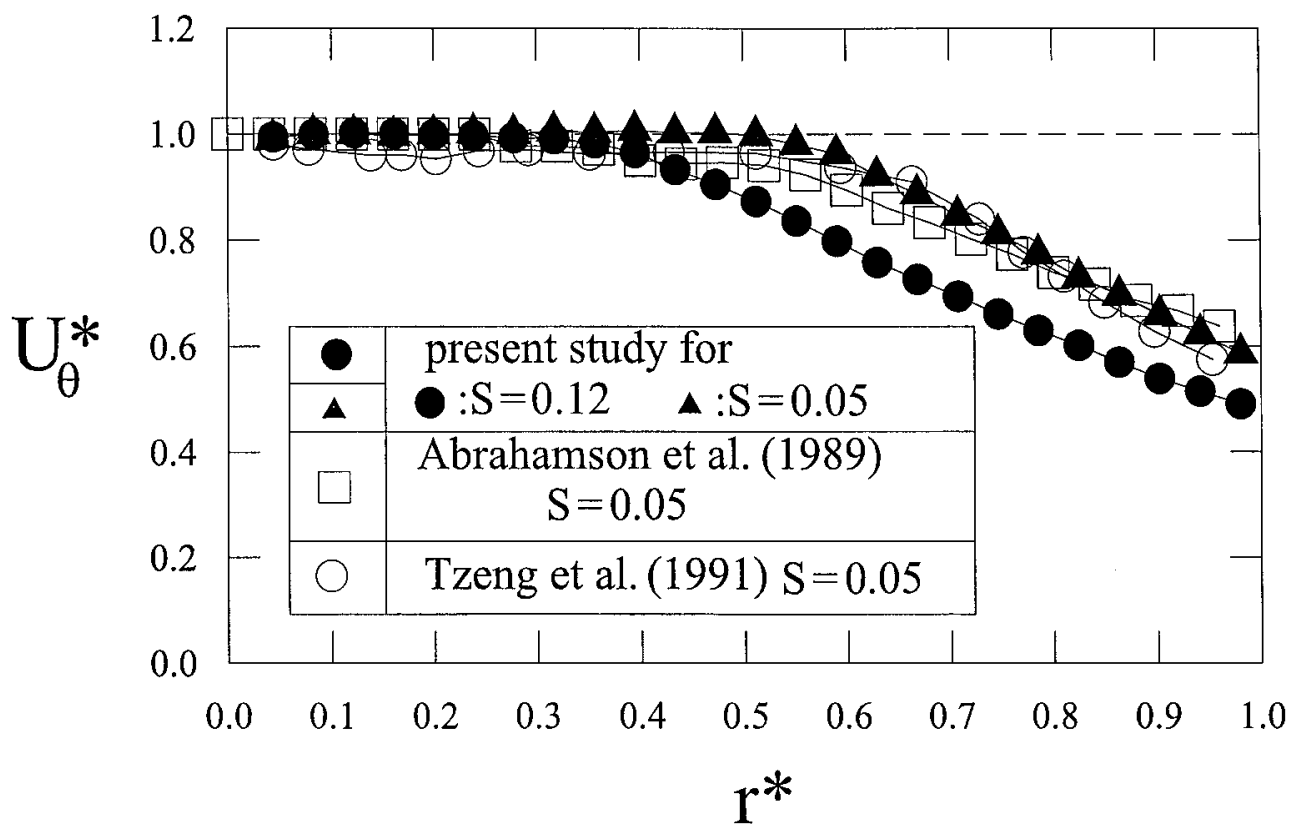

FIGURE 4

Radial profiles of the circumferential mean velocity. 


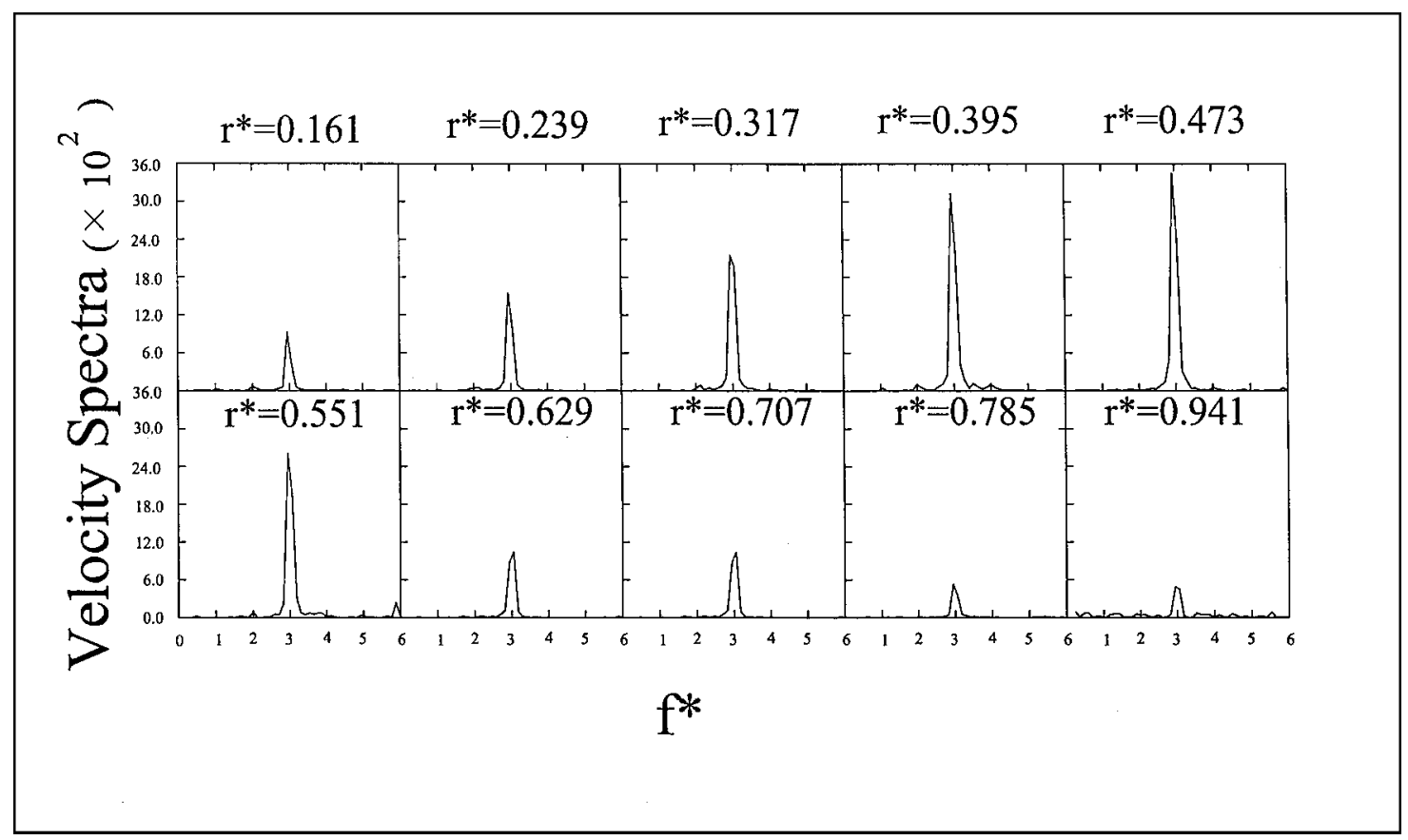

FIGURE 5

Normalized spectra of radial velocity fluctuation at various radial positions.

velocity, the spectral intensity increases with $r^{*}$ and reaches a maximum value at about $r^{*}=0.47$; it then begins to decrease to as weak a value as $r^{*} \approx 1$. The radial velocity is most energetic at $r^{*} \approx 0.5$, and its spectral intensity displays good symmetry to $r^{*} \approx 0.5$.
It is to be noted that the distribution of spectral intensity for circumferential velocity (as shown in Figure 6) is quite different from that of radial velocity. There are two local maximums of circumferential power spectra intensity. The first local maximum value takes place near the point $r^{*}=0.4$. It is also the most

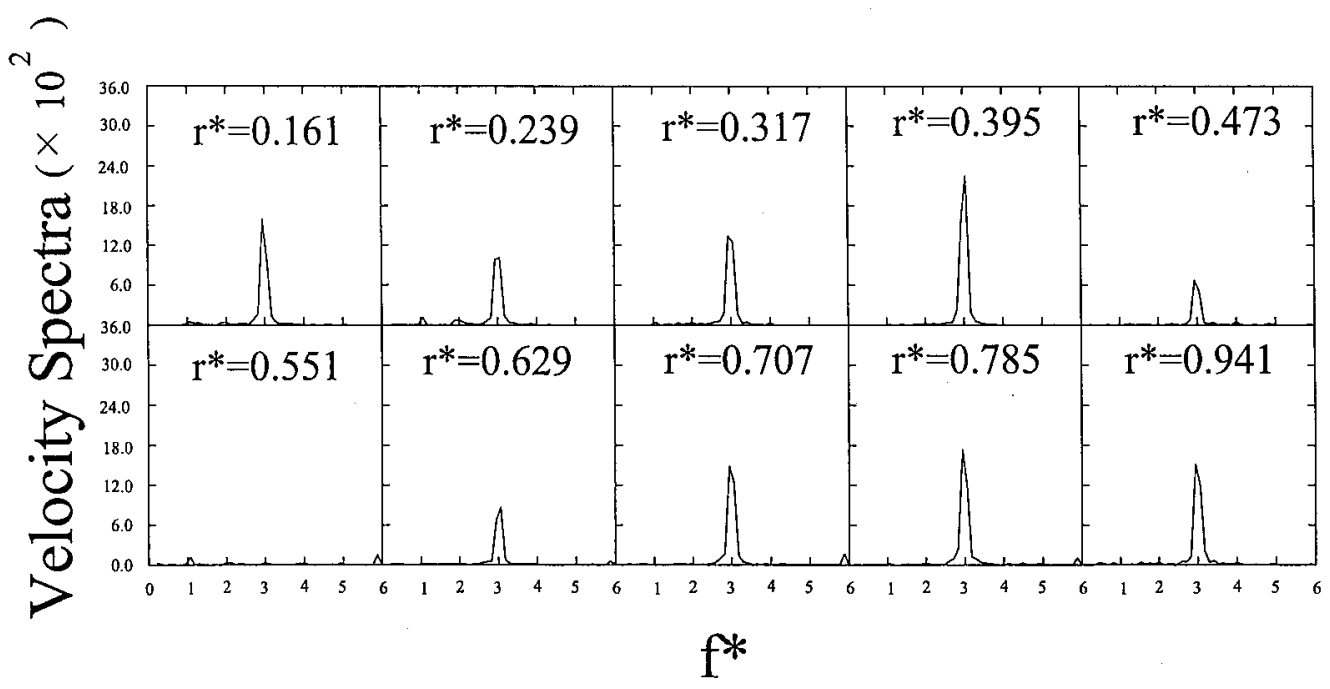

FIGURE 6

Normalized spectra of circumferential velocity fluctuation at various radial positions. 


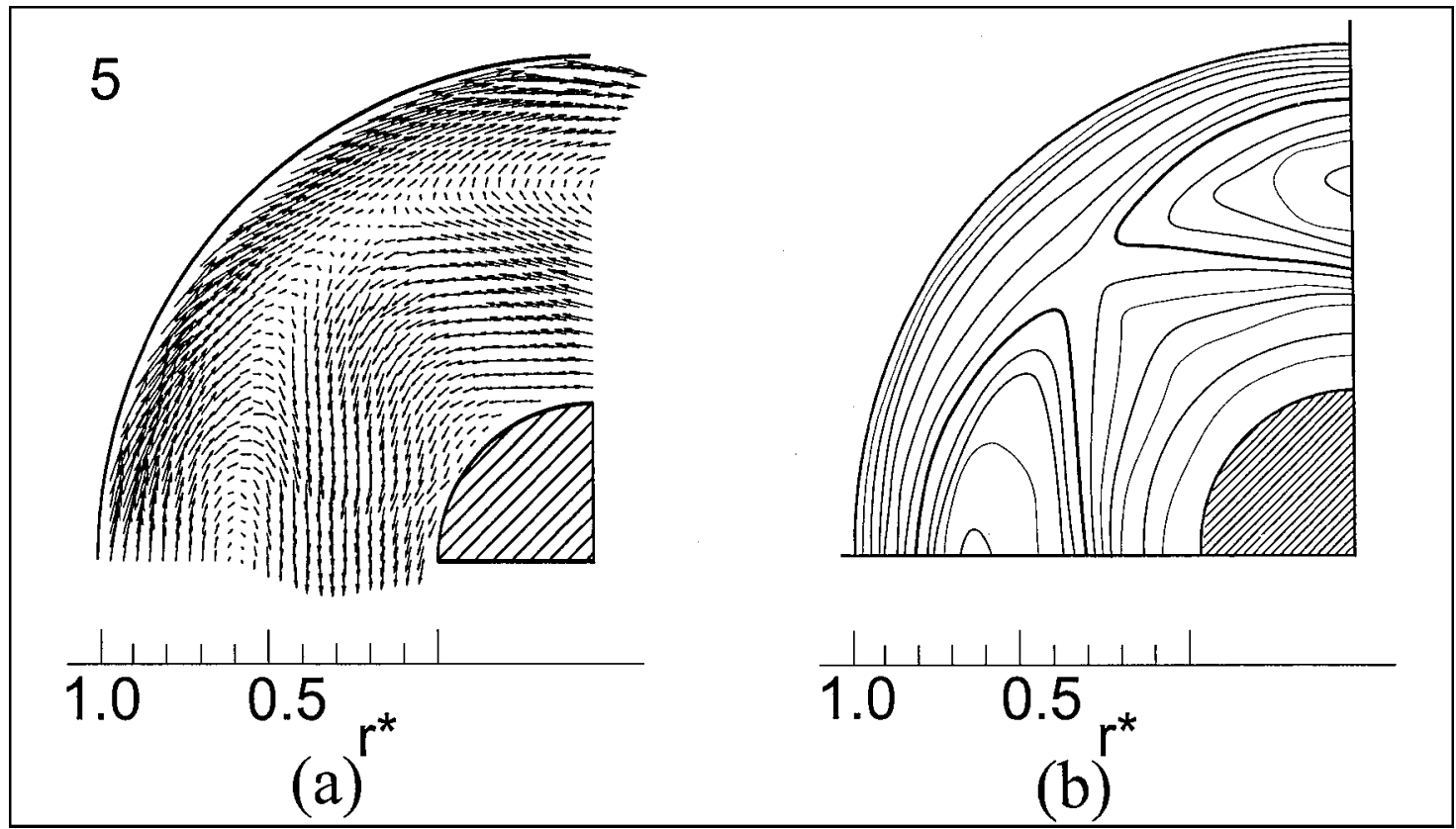

FIGURE 7

Velocity vector and streamline patterns. (a) Velocity vector, observed at a reference velocity of $0.75 \omega$. (b) Streamline pattern, observed at a reference velocity of $0.75 \omega$.

energetic spot for circumferential velocity because the highest value of the circumferential spectral intensity arises there. It recalls the point at the interface of the inner and outer regions, as discussed previously. The second local maximum occurs at $r^{*}=$ 0.72 . It is located at the center of the vortical structure. Thus, it is clear that the circumferential velocities are more energetic at the interface of the inner and outer regions and also near the center of the vortical structure.

\section{Streamline Patterns at Different Phases}

Figure $7 \mathrm{a}$ shows the vector fields, and Figure $7 \mathrm{~b}$ shows the streamline patterns for one of the 10 phases, as observed with a $0.75 \omega$ reference speed. Figure 8 shows the streamline patterns as observed with a $0.75 \omega$ reference speed. The streamline patterns at different phases during the passage of one vortex are demonstrated as an example. Some other reference speeds were also tested. The test results reveal that the vortical structure could

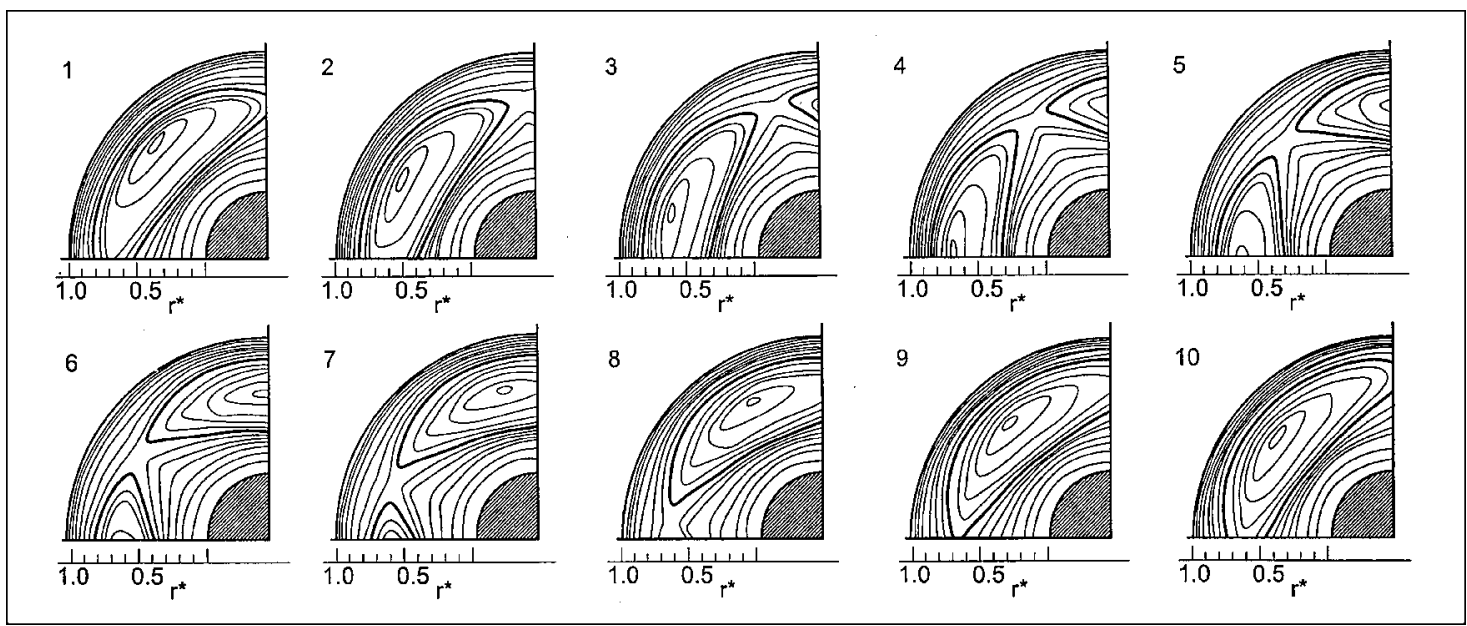

FIGURE 8

The streamline patterns at various phases at a reference velocity of $0.75 \omega$ and a contour interval of 0.2 . 
not be recognized at a higher reference speed. On the contrary, the vortical structure is located in the solid-body rotation region at a lower reference speed. This phenomenon is obviously unreasonable. The appropriate rotational speed was found to be $(0.75 \pm 0.05) \omega$ in the present case. A similar reference velocity was found by other researchers, such as Abrahamson and colleagues (1989). In their work, the camera was mounted on a motor that followed the rotation of the disks. The vortical structure could not be observed under a fixed-reference frame. Three distinct regions can be distinguished from the streamline patterns in Figure 8. The inner solid-body rotation region ranges from $r^{*} \cong 0$ to 0.35 ; the outer vortical structure region from $r^{*} \cong 0.35$ to 0.9 ; the shrouded boundary layer region from $r^{*}=0.9$ to 1 . It is noted that there seems to be no radial flow across the boundary between the rotating disks and the outsides of the disks, as shown in Figure 8. However, because the centrifugal force acts on the rotating fluid, there must exist radial inflow and outflow. The inflow and outflow regions rotate in the same direction as the disks, forming a periodic flow field (Mochizuki, 1993). Extended experiments have been carried out in the crossstream (r-z) plane (Wu, 2000). The experimental results show that there is indeed radial flow. However, the circumferential components are much larger than the radial ones, so the radial flow is not clearly observed from the phase-averaged measured data.

Although only a quarter of the frame was reconstructed, the number of the vortical structure can be easily determined to be 3 . This integer is the same as the normalized frequency found in the power spectra analysis, as shown in Figures 5 and 6 . These findings demonstrate that the velocity oscillations result from the periodic passage of flow structure. The center of the vortex is located at $r^{*}=0.72$. In addition, the radius of the inscribed circle of the vortical structure is found to be the same as that calculated by the formula $R_{i}=R_{2}\left(\frac{N-\pi A}{N+\pi A}\right)$, as proposed by Tzeng and colleagues (1991), where $\pi A=N-f_{s} / \omega$. The oscillatory frequency $f_{\mathrm{s}}$ measured is $9.9 \mathrm{~Hz}$, and the number of the vortical structure $N$ is 3 in the present study. The inscribed circle radius calculated by the formula is thereby $R_{i}=108.7 \mathrm{~mm}$. In Figure 7, the inscribed circle of each phase was drawn to find its radius, and the average value of the 10 radii was taken. The inscribed circle of the polygonal structure with the average radius intersects the dimensionless radial position at $r^{*}=\left(r-R_{1}\right) /\left(R_{2}-R_{1}\right)=0.4$, corresponding to $r=R_{i}=110.7 \mathrm{~mm}$. There is little difference between the calculated and measured values.

\section{CONCLUSIONS}

Three major conclusions may be drawn.

1. By using two laser Doppler velocimeters and the phaseaveraged method, the temporal vortical structure in the flow between corotating disks can be quantitatively reconstructed. The detailed flow information, including the mean, periodic, and random terms, provides a basis for numerical verification.
2. The power spectral analysis shows that all the peaks in the energy are at the same integral normalized frequency. The value of the normalized frequency is the same as the number of the vortical structure observed in the reconstructed streamline patterns. This indicates that the flow oscillations result from the periodic passage of the flow structure.

3. From the reconstructed streamline patterns, three distinct regions could be quantitatively identified: an inner region near the hub, an outer region, and a shroud boundary layer region.

\section{NOMENCLATURE}

a distance between disk tip and shroud

$b \quad$ thickness of disks

$f^{*} \quad$ normalized frequency

$f_{s} \quad$ dominant frequency of power spectra

$H \quad$ axial distance between disks

$N \quad$ numbers of polygonal structures

$r \quad$ radial coordinates for the system

$r^{*} \quad$ dimensionless radial coordinates, $\left(r-R_{1}\right) /\left(R_{2}-R_{1}\right)$

$R_{1} \quad$ radius of hub

$R_{2} \quad$ radius of disks

$\operatorname{Re} \quad$ Reynolds number, $\mathrm{R}_{2}^{2} \omega / \nu$

$R_{i} \quad$ radius of inscribing circle of polygon structure

$S \quad$ dimensionless axial distance of disks, $H / R_{2}$

$U, u$ velocity

$U_{r} \quad$ radial velocity component

$U_{\text {ref }}$ circumferential velocity of the reference point

$U_{\theta} \quad$ circumferential velocity component

$U_{\theta}^{*} \quad$ normalized circumferential velocity, $U_{\theta} / r \omega$

$\bar{u} \quad$ global mean of $u$

$\tilde{u} \quad$ periodic fluctuation away from $\bar{u}$

$u^{\prime} \quad$ random fluctuation away from $\tilde{u}$

$Z$ axial coordinates of the system

\section{Greek Letters}

$\theta \quad$ circumferential coordinates for the system

$v \quad$ kinematic viscosity

$\omega \quad$ rotating speed of disks

\section{REFERENCES}

Abrahamson, S. D., Koga, D. J., and Eaton, J. K. 1988. Flow visualization and spectral measurements in a simulated rigid disk drive. IEEE Trans. Comp., Hybrids and Manuf. Tech. 11:576-584.

Abrahamson, S. D., Eaton, J. K., and Koga, D. J. 1989. The flow between shrouded co-rotating disks. Phys. Fluids A 1:241-251.

Abrahamson, S. D., Eaton, J. K., and Chang, C. 1991. Flow structure in head-disk assemblies and implications for design. Adv. Info. Storage Syst. 1:111-132.

Durst, F., Melling, A., and Whitelaw, J. H. 1981. Principles and Practice of Laser-Doppler Anemometry. London: Academic.

Kaneko, R., Oguchi, S., and Hoshiya, K. 1977. Hydrodynamic characteristics in disk packs for magnetic storage. Rev. Elec. Comm. Lab. 25:1325-1336.

Lennemann, E. 1974. Aerodynamic aspects of disk files. IBM J. Res. Develop 18:480-488. 
McLaughlin, D. K., and Tiederman, W. G. 1973. Biasing correction for individual realization of laser anemometer measurements in turbulent flows. Phys. Fluids 16:2082-2088.

Mochizuki, S. 1993. Unsteady flow phenomena and heat transfer in rotating-disk systems. Proceedings of the 6th International Symposium on Transport Phenomena in Thermal Engineering 279-289. Seoul, Korea.

Humphrey, J. A. C., and Gor, D. 1993. Experimental observation of an unsteady detached shear layer in enclosed co-rotating disk flow. Phys. Fluids 5:2438.

Humphrey, J. A. C., Schuler, C. A., and Weber, D. R. 1995. Unsteady laminar flow between a pair of disks co-rotating in a fixed cylindrical enclosure. Phys. Fluids 7:1225-1240.

Hussain, A. K. M. F., and Reynolds, W. C. 1970. The mechanics of an organized wave in turbulent shear flow. J. Fluid Mech. 41:241-258.
Reynolds, W. C., and Hussain, A. K. M. F. 1972. The mechanics of an organized wave in turbulent shear flow. 3: theoretical models and comparisons with experiments. J. Fluid Mech. 54:263-288.

Schuler, C. A., Usry, W., Weber, B., Humphrey, J. A. C., and Greif, R. 1990. On the flow in the unobstructed space between shrouded co-rotating disks. Phys. Fluids A 2:1760-1770.

Tzeng, H. M., Munce, A. C., and Crawforth, L. 1991. Quantitative airflow visualization between shrouded co-rotating disks. ASME Exp. Num. Flow Visualization 128:141-147.

Tzeng, H. M., and Humphrey, J. A. C. 1991. Co-rotating disk flow in an axisymmetric enclosure with and without a bluff-body. Int. J. Head and Fluid Flow 12:194-201.

Wu, S. C. 2000. The Flow Between Corotating Disks With/Without an Obstruction in a Cylindrical Enclosure (PhD diss, National Taiwan University). 

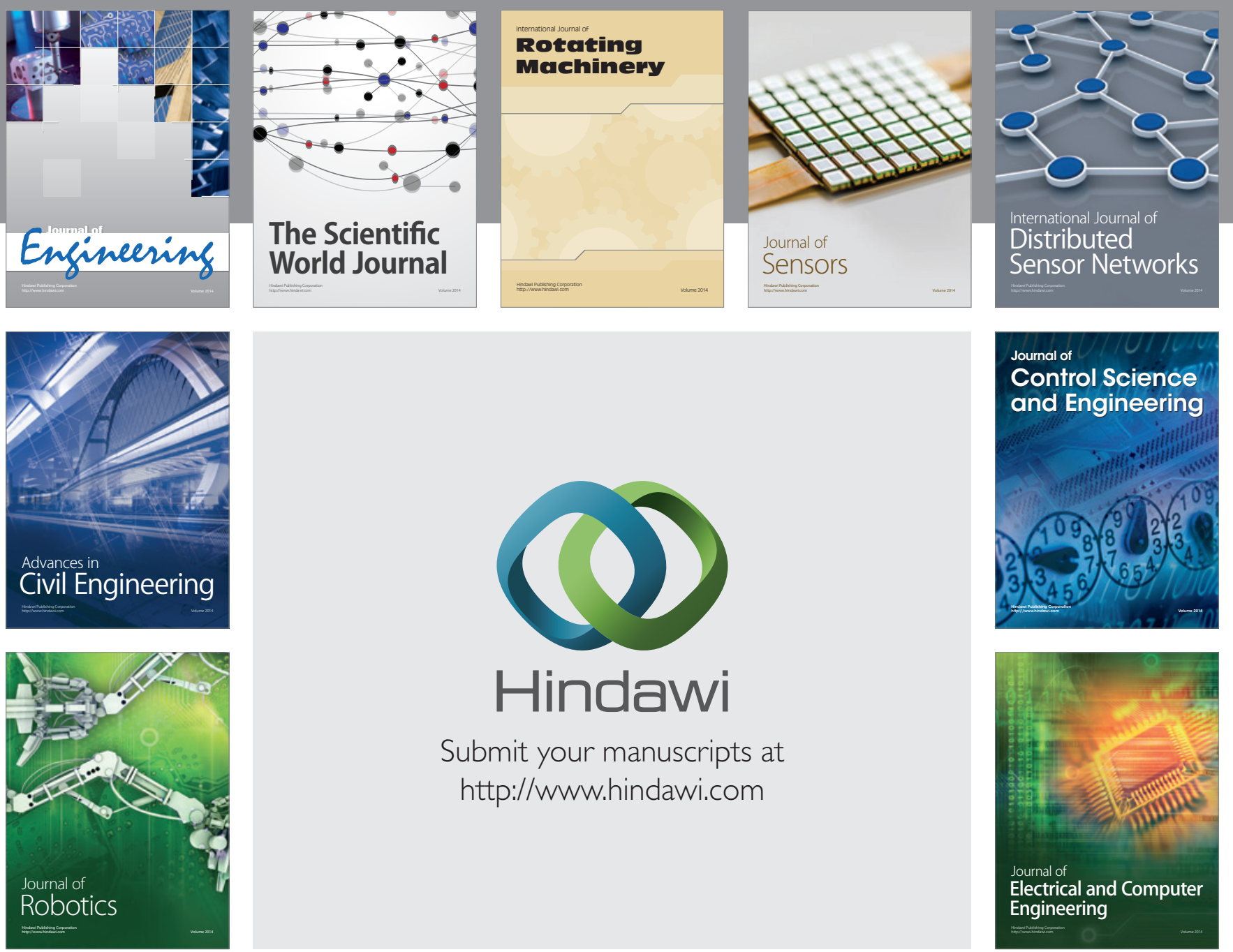

Submit your manuscripts at

http://www.hindawi.com
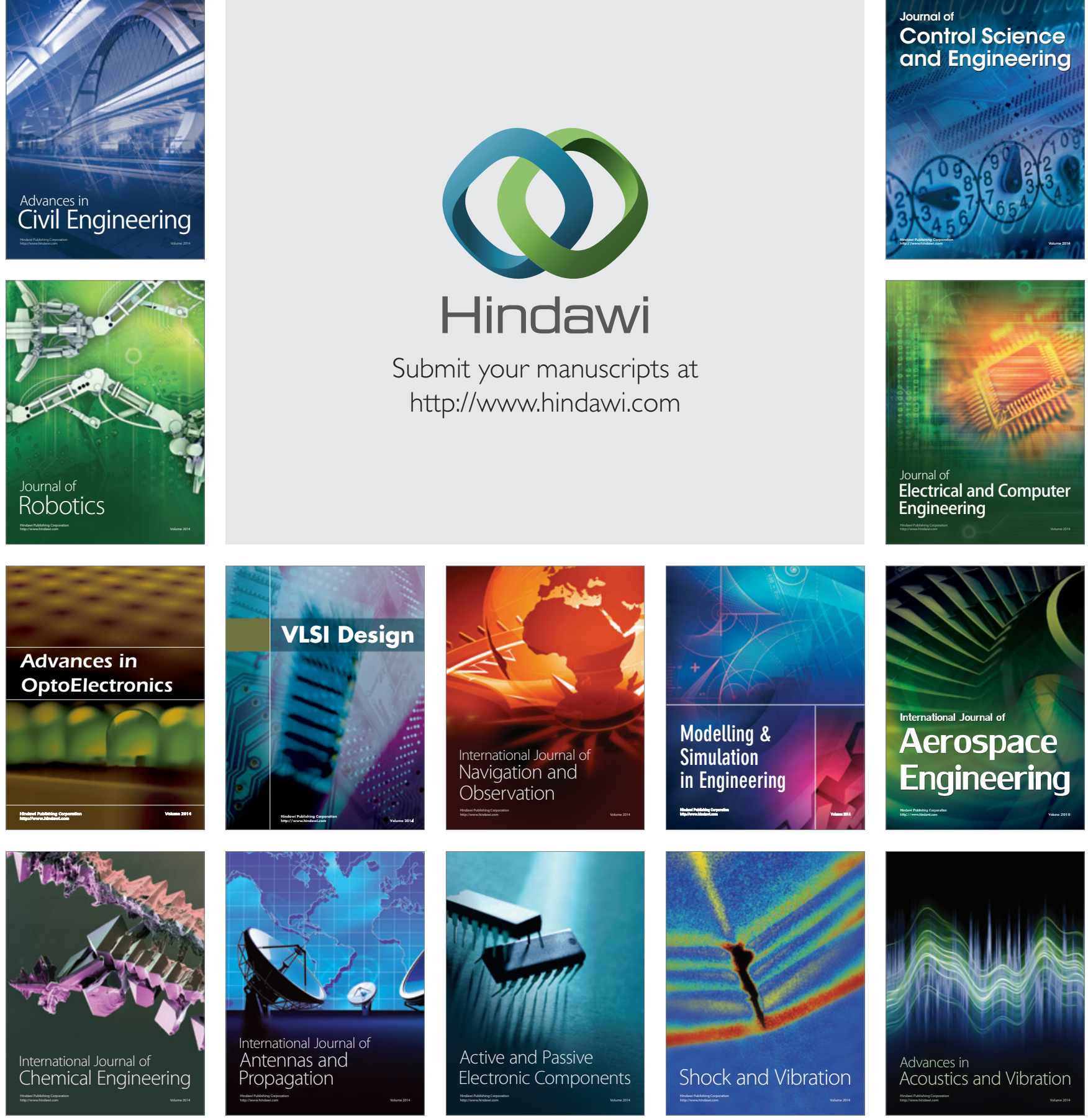